\title{
Synthesis and Antimicrobial Activities of Some New Heterocyclic Compounds Based on 6-Chloropyridazine-3(2H)-thione
}

\author{
Nasser M. Abd El-Salam, ${ }^{1}$ Mohamed S. Mostafa, \\ Gamal A. Ahmed, ${ }^{3}$ and Othman Y. Alothman ${ }^{4}$ \\ ${ }^{1}$ Riyadh Community College, King Saud University, P.O. Box 28095, Riyadh 11437, Saudi Arabia \\ ${ }^{2}$ Chemistry Department, Faculty of Science, Jazan University, P.O. Box 2079, Jazan, Saudi Arabia \\ ${ }^{3}$ Chemistry Department, Faculty of Science, Zagazig University, Zagazig 44519, Egypt \\ ${ }^{4}$ Chemical Engineering Department, College of Engineering, King Saud University, P.O. Box 800, Riyadh 11421, Saudi Arabia
}

Correspondence should be addressed to Nasser M. Abd El-Salam; nelsalam@ksu.edu.sa

Received 21 January 2013; Revised 27 February 2013; Accepted 28 February 2013

Academic Editor: Patricia Valentao

Copyright (C) 2013 Nasser M. Abd El-Salam et al. This is an open access article distributed under the Creative Commons Attribution License, which permits unrestricted use, distribution, and reproduction in any medium, provided the original work is properly cited.

\begin{abstract}
Three tricyclic ring system, pyridazino[6,1-b]quinazolin-10-ones, benzimidazolo-pyridazine thione, and 1,2,4-benzotriazinopyridazinethione along with imidazo-[1,2-b]-pyridazinethione, 1,2,4-triazolo[4,3-b]pyridazine-thione derivatives were synthesized starting from 6-chloropyridazin3- $(2 \mathrm{H})$-thione. Some disulfide and sulfide derivatives were also prepared. The antimicrobial activity of the synthesized compounds was tested. Some of these compounds possess a highly response against gram-positive and gramnegative bacteria as well as fungi.
\end{abstract}

\section{Introduction}

The chemistry of pyridazines and their fused heterocyclic derivatives has received considerable attention owing to their synthetic and effective biological importance. Pyridazines have been reported to possess antimicrobial [1-3], antituberculosis [4-6], antifungal [7], anticancer [8], antihypertensive [9], herbicidal [10], anti-inflammatory [11] activities, and protein tyrosine phosphatase $1 \mathrm{~B}$ (PTP1B) inhibitors [12]. They also have an immense potential in agricultural science as plant growth regulators and crop protection agents [13]. The incorporation of two moieties increases biological activity of both and thus it was of value to synthesize some new heterocyclic derivatives having two moieties in the same molecules. Several derivatives of pyridazine incorporating 1,2,4-triazole, imidazole, isoxazole, and triazine rings have been shown to display a wide spectrum in biological and therapeutic areas [14-18]. Prompted by these observations and in continuation to authors' work on the synthesis of new pyridazine compounds $[19,20]$, authors report herein the use of 6-chloropyridazin-3(2H)-thione (1) for the synthesis of new pyridazine compounds with the aim to evaluate their antimicrobial activities.

\section{Experimental}

Uncorrected melting points were determined on Gallenkamp Electric Melting point Apparatus. IR spectra ( $\mathrm{KBr}$ Disces) were recorded on a FT/IR-400 Spectrophotometer (Perkin Elmer). ${ }^{1} \mathrm{H}$ NMR $(300 \mathrm{MHz})$ and ${ }^{13} \mathrm{C}$ NMR $(75 \mathrm{MHz})$ spectra were recorded on a Varian instrument. Chemical shifts were reported as $\delta$ values relative to tetramethylsilane (TMS) as internal reference. Elemental analyses for $\mathrm{C}, \mathrm{H}, \mathrm{N}$, and $\mathrm{S}$ were performed on a Perkin-Elmer 240 microanalyser in the Faculty of Science, Cairo University.

2.1. Reaction of 6-Chloropyridazin-3(2H)-thione (1) with Anthranilic Acid Derivatives, o-Aminophenol, and o-Chlorophenylhydrazine: Formation of 2-Thioxo-1,2,10-trihydropyridazino[6,1-b]quinazolin-10-ones (2a-c), Benzimidazolo[2,3-a]pyridazine-3(2H)-thione (3), and 1, 2,4-Benzotriazino[3,4-a]pyridazine-3(2H)-thione (4)

General Procedure. To a solution of compound (1) (0.01 mole) in absolute ethanol $(30 \mathrm{~mL})$, equimolar amounts of anthranilic acid derivatives (namely, anthranilic acid, 


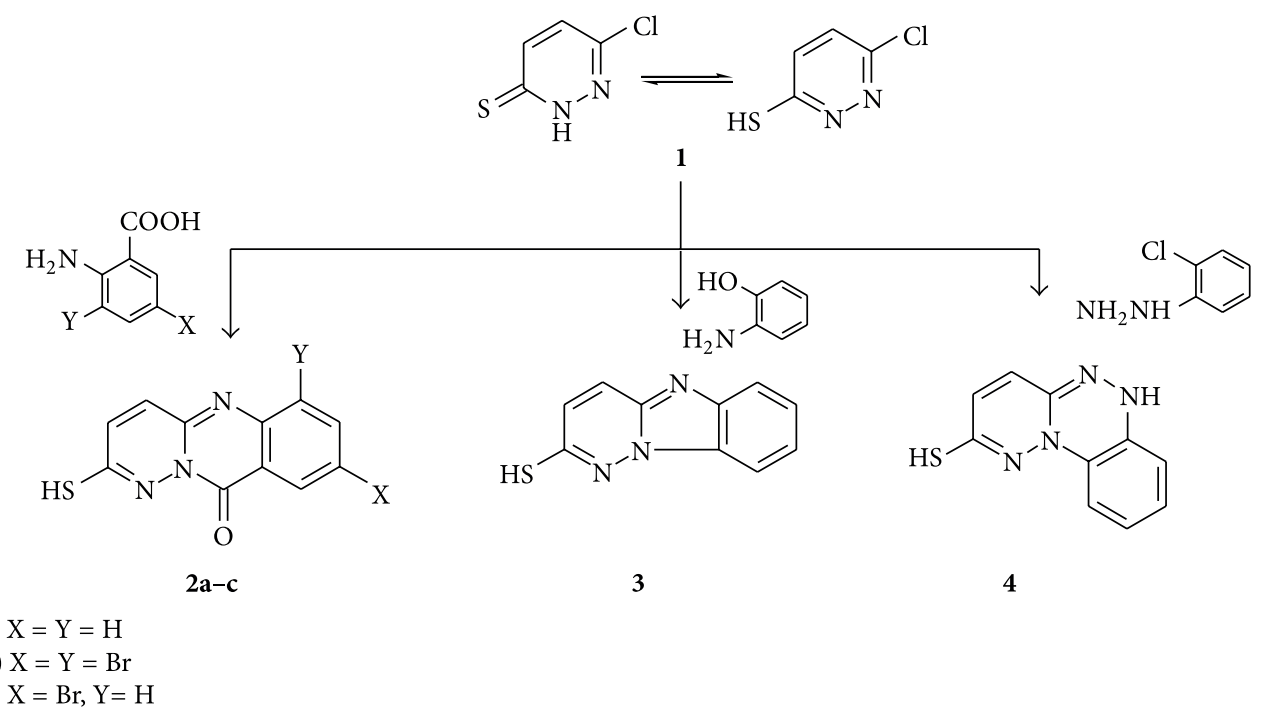<smiles>NNc1ccccc1Cl</smiles><smiles>NNc1ccccc1Cl</smiles><smiles></smiles><smiles>SC1=NN2C(=NNc3ccccc32)C=C1</smiles>

SCHEME 1

3,5-dibromoanthranilic acid, and 5-bromo-anthranilic acid), $o$-aminophenol, and $o$-chlorophenylhydrazine were added and the reaction mixture was refluxed for $7-8 \mathrm{~h}$. The solid product, obtained upon cooling, was filtered off, dried, and crystallized from an appropriate solvent to give compounds $(\mathbf{2 a}-\mathbf{c}),(3)$, and (4), respectively, shown in Scheme 1 (Tables 2 and 3$)$.

2.2. Reaction of (1) with Phenylalanine: Formation of Imidazo [1,2-b]pyridazine-3(2H)-thione Derivative (5). A mixture of 1 ( 0.01 mole) and phenylalanine $(0.01$ mole $)$ in butanol $(20 \mathrm{~mL})$ was heated under reflux for $6 \mathrm{~h}$. The solvent was concentrated under vacuum and allowed to cool. The separated solid was filtered off and recrystallized from ethanol to afford (5), shown in Scheme 2 (Tables 2 and 3).

2.3. Reaction of (1) with Thiosemicarbazide and Benzoylhydrazines: Formation of 1,2,4-triazolo[4,3-b]pyridazinethione Derivatives (6) and $(7 \boldsymbol{a}, \boldsymbol{b})$. A solution of $\mathbf{1}(0.01 \mathrm{~mole})$ in ethanol $(30 \mathrm{~mL})$ was treated with thiosemicarbazide or benzoylhydrazines ( 0.01 mole for each) and refluxed for $6 \mathrm{~h}$. The solution was concentrated and cooled, the solid, so separated, was collected and recrystallized from suitable solvent to yield 6 and $7 \mathbf{a}, \mathbf{b}$ (Tables 2 and 3 ).

2.4. Reaction of (1) with Acetophenonehydrazone Derivatives: Formation of Compounds $(\mathbf{8} \boldsymbol{a}, \boldsymbol{b})$. A mixture of (1) $(0.01 \mathrm{~mol})$ and acetophenonehydrazone and/or 3-nitroacetophenonehydrazone $(0.01 \mathrm{~mol})$, was fused on oil bath at $180^{\circ} \mathrm{C}$ for $2 \mathrm{~h}$. The solid mass obtained upon air cooling was refluxed with ethanol $(20 \mathrm{~mL})$ for $2 \mathrm{hr}$. The precipitate obtained upon cooling was collected and crystallized from methanol into (8a, b) (Tables 2 and 3).

2.5. Reaction of (1) with p-Toluenesulfonyl Hydrazine: Formation of (9). A mixture of compound (1), (0.01 mol) and $p$ toluenesulfonylhydrazine $(0.01 \mathrm{~mol})$ in butanol $(30 \mathrm{~mL})$ was 


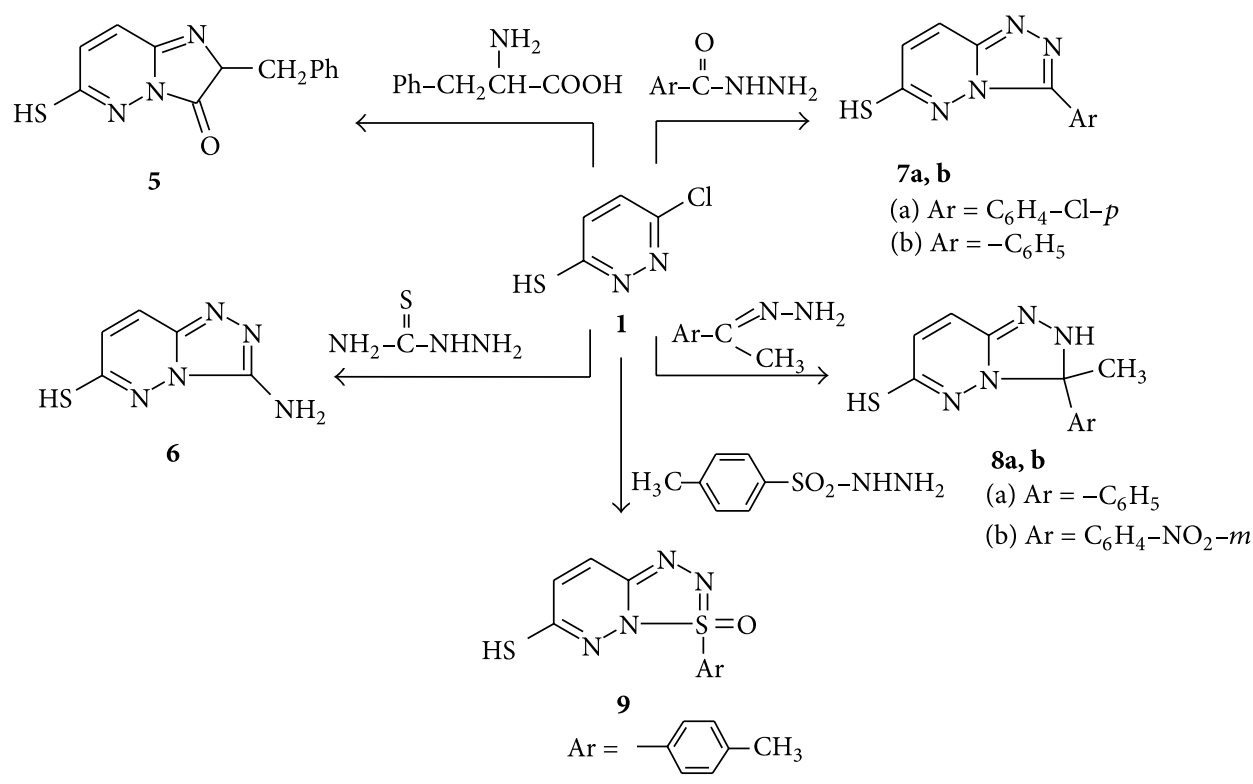

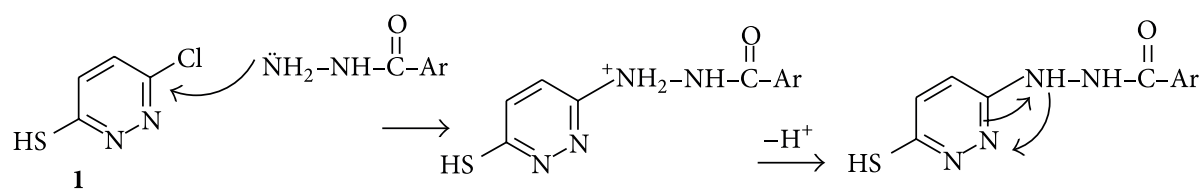

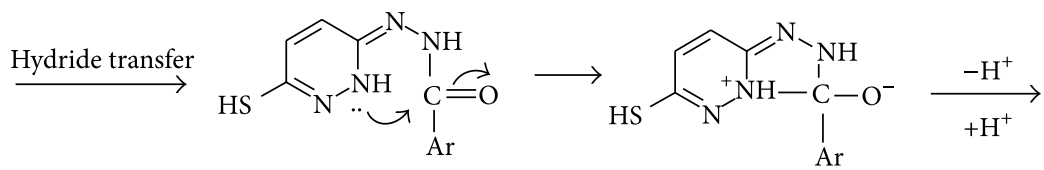<smiles>[Y14]c1nnc2ccc(S)nn12</smiles>

SCHEME 2

heated under reflux for $6 \mathrm{~h}$. The reaction mixture was left to cool at room temperature and the solid formed was collected and crystallized from ethanol to give $\mathbf{9}$ (Tables 2 and 3 ).

2.6. Formation of 2,4-Dinitrophenyl-6-chloropyridazyldisulfide (10). Compound 1 (0.01 mole) was stirred at room temperature for $1 \mathrm{~h}$ with an equimolar amount of 2,4dinitrobenzensulfenyl chloride in acetic acid $(25 \mathrm{~mL})$. The reaction mixture was then concentrated by evaporation and the product was crystallized from methanol to produce $\mathbf{1 0}$ (Tables 2 and 3 ).

2.7. Formation of Bis(6-chloropyridazyl)disulfide (11). A solution of (1) (0.01 mole) was left at room temperature for $4 \mathrm{~h}$ in acetic acid $(15 \mathrm{~mL})$ and sodium nitrite $(0.01 \mathrm{~mol})$, then poured in cold water $(20 \mathrm{~mL})$. The obtained solid was collected and crystallized from mixture of petroleum ether $60-80$ and benzene, to give (11) (Tables 2 and 3).

2.8. Reaction of (1) with Thiophenol or 3,6-Dichloropyridazine: Formation of Phenyl pyridazyl-thione disulfide (12) and Bis(6-chloropyridazyl)sulfide (13). A mixture of compound (1), (0.01 mole) and thiophenol or 3,6-dichloropyridazine (0.01 mole for each) was refluxed in ethanol $(30 \mathrm{~mL})$ containing sodium metal $(0.01 \mathrm{~mol})$, for $8 \mathrm{~h}$. The reaction mixture was left to cool; the solid produced was collected and crystallized from proper solvent to yield (12) and (13) (Tables 2 and 3).

\section{Results and Discussion}

6-Chloropyridazin-3(2H)-thione (1) has been used as the key starting material for the preparation of some new 
TABLE 1: Antimicrobial activity of synthetic compounds.

\begin{tabular}{|c|c|c|c|c|c|c|}
\hline \multirow{2}{*}{ Compd. no. } & \multicolumn{2}{|c|}{ Gram +ve Bacteria } & \multicolumn{2}{|c|}{ Gram -ve bacteria } & \multicolumn{2}{|c|}{ Fungi } \\
\hline & S. aureus & B. subtilis & P. aurignosa & E. coli & C. albicans & A. niger \\
\hline $2 a$ & 24 & 30 & 22 & 20 & 19 & 14 \\
\hline $2 b$ & 18 & 20 & 10 & 10 & 10 & 13 \\
\hline $2 c$ & 18 & 10 & 11 & 10 & 16 & 12 \\
\hline 3 & 20 & 23 & 17 & 17 & 20 & 15 \\
\hline 4 & 25 & 27 & 11 & 12 & 19 & 14 \\
\hline 5 & 18 & 19 & 10 & 18 & 18 & 15 \\
\hline 6 & 21 & 23 & 16 & 12 & 18 & 14 \\
\hline $7 \mathbf{a}$ & 12 & 20 & 19 & 17 & 16 & 12 \\
\hline $7 b$ & 11 & 16 & 11 & 18 & 19 & 13 \\
\hline $8 \mathbf{a}$ & 15 & 13 & 14 & 11 & 22 & 16 \\
\hline $8 b$ & 19 & 22 & 10 & 12 & 19 & 15 \\
\hline 9 & 26 & 29 & 22 & 20 & 23 & 18 \\
\hline 10 & 17 & 16 & 15 & 13 & 18 & 12 \\
\hline 11 & 19 & 19 & 22 & 20 & 16 & 15 \\
\hline 12 & 14 & 19 & 18 & 17 & 24 & 17 \\
\hline 13 & 14 & 11 & 13 & 15 & 19 & 10 \\
\hline Ampicillin & 22 & 26 & 20 & 19 & - & - \\
\hline Micostatin & - & - & - & - & 22 & 16 \\
\hline
\end{tabular}

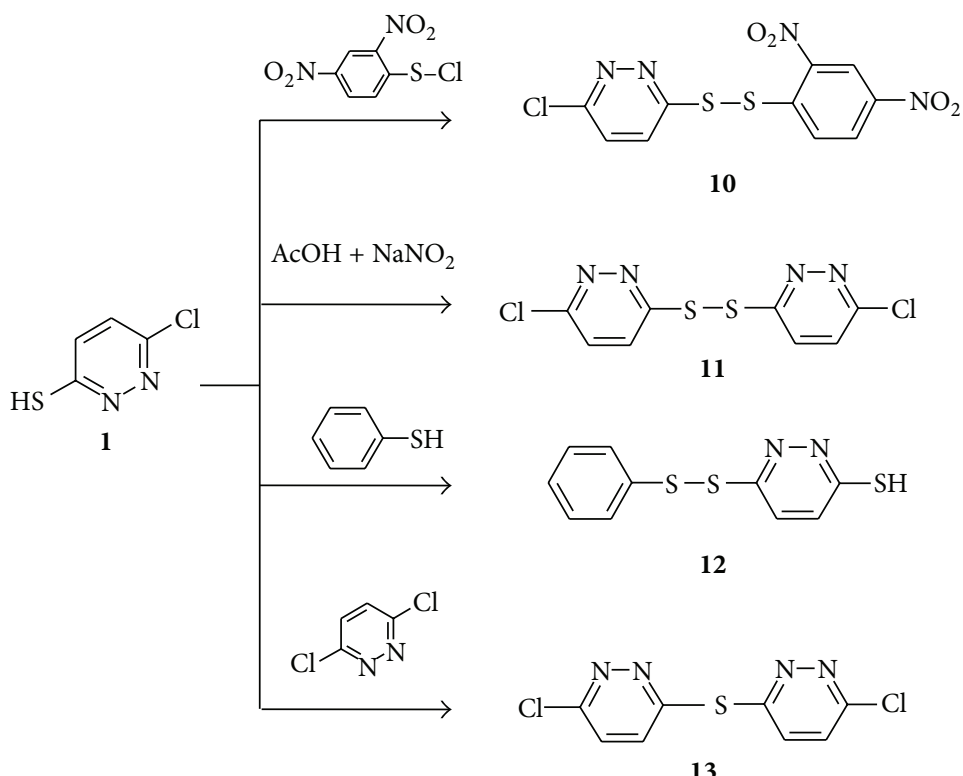

SCHEMe 3

heterocyclic compounds. Thus, (1) reacts with bifunctional nucleophile like anthranilic acid, 2-aminophenol, and 2chlorophenylhydrazine to afford a tricyclic ring system as 2-thioxo-1,2,10-trihydropyridazino[6,1-b] quinazolin-10-ones $(\mathbf{a} \mathbf{a}-\mathbf{c})$, benzimidazolopyridazine thione (3), and 1,2,4benzotriazinopyridazinethione (4) derivatives, respectively (Scheme 1). The IR spectrum of $(\mathbf{2} \mathbf{a}-\mathbf{c})$ showed characteristic absorption bands for (NH) at 3410-3380, (CO) at 1750-1616 and $(\mathrm{C}=\mathrm{N})$ at $1620-1580 \mathrm{~cm}^{-1}$, while that of (3) and (4) showed the bands of $(\mathrm{NH})$ at 3370-3317 and $(\mathrm{C}=\mathrm{N})$ at $1610-1590 \mathrm{~cm}^{-1}$ (Tables 1 and 2).

Imidazo[1,2-b]pyridazinethione derivative (5) was prepared by treatment compound (1) with phenylalanine in refluxing butanol (Scheme 2). The IR spectrum of 5 revealed absorption bands at $3310(\mathrm{NH}), 1660$ (CO), and $1605 \mathrm{~cm}^{-1}$ $(\mathrm{C}=\mathrm{N})$. In the ${ }^{1} \mathrm{H} \mathrm{NMR}$ of (5), the two doublet signals 
TABle 2: Physical properties of the prepared compounds (2-13).

\begin{tabular}{|c|c|c|c|c|c|c|c|c|}
\hline \multirow{2}{*}{ Compd. no. } & \multirow{2}{*}{ m.p. ${ }^{\circ} \mathrm{C}$} & \multirow{2}{*}{ Yield (\%) } & \multirow{2}{*}{ Solvent of cryst. } & \multirow{2}{*}{ Mol. formula (M.wt) } & \multicolumn{4}{|c|}{ Elemental analysis Calcd./Found } \\
\hline & & & & & $\mathrm{C}$ & $\mathrm{H}$ & $\mathrm{N}$ & S \\
\hline \multirow{2}{*}{$2 a$} & \multirow{2}{*}{$182-184$} & \multirow{2}{*}{37} & \multirow{2}{*}{ Ethanol } & $\mathrm{C}_{11} \mathrm{H}_{7} \mathrm{~N}_{3} \mathrm{OS}$ & 57.61 & 3.10 & 18.35 & 13.99 \\
\hline & & & & 229.26 & 57.63 & 3.08 & 18.33 & 13.98 \\
\hline \multirow{2}{*}{$2 b$} & \multirow{2}{*}{$255-257$} & \multirow{2}{*}{41} & \multirow{2}{*}{ Methanol } & $\mathrm{C}_{11} \mathrm{H}_{5} \mathrm{Br}_{2} \mathrm{~N}_{3} \mathrm{OS}$ & 34.16 & 1.33 & 10.89 & 8.30 \\
\hline & & & & 387.05 & 34.14 & 1.30 & 10.86 & 8.28 \\
\hline \multirow{2}{*}{$2 c$} & \multirow{2}{*}{$232-234$} & \multirow{2}{*}{45} & \multirow{2}{*}{ Ethanol } & $\mathrm{C}_{11} \mathrm{H}_{6} \mathrm{BrN}_{3} \mathrm{OS}$ & 42.91 & 1.98 & 13.68 & 10.42 \\
\hline & & & & 308.15 & 42.88 & 1.96 & 13.64 & 10.40 \\
\hline \multirow{2}{*}{3} & \multirow{2}{*}{$168-170$} & \multirow{2}{*}{49} & \multirow{2}{*}{ Methanol } & $\mathrm{C}_{10} \mathrm{H}_{7} \mathrm{~N}_{3} \mathrm{~S}$ & 59.70 & 3.56 & 20.92 & 15.96 \\
\hline & & & & 201.25 & 59.68 & 3.51 & 20.88 & 15.93 \\
\hline \multirow{2}{*}{4} & \multirow{2}{*}{$285-287$} & \multirow{2}{*}{39} & \multirow{2}{*}{ Acetic acid } & $\mathrm{C}_{10} \mathrm{H}_{8} \mathrm{~N}_{4} \mathrm{~S}$ & 55.56 & 3.92 & 25.95 & 14.92 \\
\hline & & & & 216.26 & 55.54 & 3.73 & 25.91 & 14.82 \\
\hline \multirow{2}{*}{5} & \multirow{2}{*}{$282-283$} & 61 & Ethanol & $\mathrm{C}_{13} \mathrm{H}_{11} \mathrm{~N}_{3} \mathrm{OS}$ & 60.58 & 4.78 & 16.38 & 12.49 \\
\hline & & 61 & Etnanor & 257.06 & 60.68 & 4.31 & 16.33 & 12.46 \\
\hline 6 & $279-280$ & 58 & Ethanol & $\mathrm{C}_{5} \mathrm{H}_{5} \mathrm{~N}_{5} \mathrm{~S}$ & 35.94 & 3.07 & 41.92 & 19.20 \\
\hline & & & & 167.19 & 35.92 & 3.01 & 41.89 & 19.18 \\
\hline $7 \mathbf{a}$ & $261-263$ & 52 & Ethanol & $\mathrm{C}_{11} \mathrm{H}_{7} \mathrm{ClN}_{4} \mathrm{~S}$ & 50.33 & 2.71 & 21.37 & 12.24 \\
\hline ta & $201-200$ & $J 2$ & 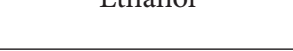 & 262.72 & 50.29 & 2.69 & 21.33 & 12.20 \\
\hline $7 \mathbf{b}$ & $242-243$ & 63 & Ethanol & $\mathrm{C}_{11} \mathrm{H}_{8} \mathrm{~N}_{4} \mathrm{~S}$ & 57.85 & 3.55 & 24.56 & 14.07 \\
\hline $7 \mathrm{~b}$ & $242-243$ & 63 & Etnanor & 228.27 & 57.88 & 3.53 & 24.54 & 14.04 \\
\hline $8 \mathbf{a}$ & $271-273$ & 35 & Methanol & $\mathrm{C}_{12} \mathrm{H}_{12} \mathrm{~N}_{4} \mathrm{~S}$ & 59.02 & 4.99 & 22.97 & 13.10 \\
\hline & & & & 244.31 & 58.99 & 4.95 & 22.93 & 13.12 \\
\hline $8 b$ & $246-248$ & 44 & Methanol & $\mathrm{C}_{12} \mathrm{H}_{11} \mathrm{~N}_{5} \mathrm{O}_{2} \mathrm{~S}$ & 49.78 & 3.86 & 24.23 & 11.12 \\
\hline ov & $240-240$ & 44 & victiranot & 289.31 & 49.82 & 3.83 & 24.21 & 11.08 \\
\hline 9 & $205-207$ & 49 & Ethanol & $\mathrm{C}_{11} \mathrm{H}_{10} \mathrm{~N}_{4} \mathrm{OS}_{2}$ & 47.52 & 3.57 & 20.17 & 23.09 \\
\hline 9 & $205-207$ & 49 & Etnanor & 278.35 & 47.47 & 3.62 & 20.13 & 23.04 \\
\hline 10 & $239-240$ & 73 & Methanol & $\mathrm{C}_{10} \mathrm{H}_{5} \mathrm{ClN}_{4} \mathrm{O}_{4} \mathrm{~S}_{2}$ & 34.86 & 1.62 & 16.29 & 18.63 \\
\hline & & & & 344.75 & 34.84 & 1.46 & 16.25 & 18.60 \\
\hline 11 & $221-223$ & 85 & ether $60-80 /$ Benzene & $\mathrm{C}_{8} \mathrm{H}_{4} \mathrm{Cl}_{2} \mathrm{~N}_{4} \mathrm{~S}_{2}$ & 33.04 & 1.42 & 19.25 & 22.09 \\
\hline iI & $221-223$ & ou & Cथात & 291.17 & 33.00 & 1.38 & 19.24 & 22.02 \\
\hline 12 & $234-236$ & 73 & Ethanol & $\mathrm{C}_{10} \mathrm{H}_{8} \mathrm{~N}_{2} \mathrm{~S}_{3}$ & 47.64 & 3.25 & 11.14 & 38.15 \\
\hline 12 & $234-236$ & 13 & Ethanol & 252.37 & 47.59 & 3.20 & 11.10 & 38.11 \\
\hline 13 & $186-88$ & 71 & Methanol & $\mathrm{C}_{8} \mathrm{H}_{4} \mathrm{Cl}_{2} \mathrm{~N}_{4} \mathrm{~S}$ & 37.10 & 1.59 & 21.65 & 12.40 \\
\hline & & & & 259.11 & 37.08 & 1.56 & 21.62 & 12.37 \\
\hline
\end{tabular}

appeared at 2.95 and $3.15 \mathrm{ppm}$ for $\left(-\mathrm{CH}_{2}\right)$ group, while $\mathrm{NH}$ and $\mathrm{Ar}-\mathrm{H}$ peaks were observed at 6.83 and $7.41-7.96 \mathrm{ppm}$, respectively.

Treatment of 6-chloro-pyrizadinethione (1) with thiosemicarbazide or benzoylhydrazines in boiling ethanol and by fusion with acetophenone hydrazones afforded 1,2,4triazolo[4,3-b]pyridazinethione derivatives $(6),(7 \mathbf{a}, \mathbf{b})$, and $(\mathbf{8 a}, \mathbf{b})$, respectively (Scheme 2$)$. The IR spectra of these triazolopyridazine derivatives showed absorption bands at 3420$3349 \mathrm{~cm}^{-1}$ for $\mathrm{NH}$; furthermore, compound (6) exhibited a characteristic band for amino group. The ${ }^{1} \mathrm{H}$ NMR spectrum of compound (6) displayed two exchangeable $\left(\mathrm{NH}_{2}\right)$ protons as a singlet signal at $6.29 \mathrm{ppm}$ (Table 2). On the other hand, compound (1) reacted with $p$-toluenesulfonyl hydrazine to give compound (9).
Finally, disulfide derivatives were prepared. Stirring a solution of pyridazine-3-thione (1) and 2,4-dinitrobenzenesulfenyl chloride in acetic acid at room temperature afforded 2,4-dinitrophenyl-6-chloropyridazyldisulfide (10) (Scheme 3). The ${ }^{1} \mathrm{H}$ NMR of this disulfide exhibited signals at $\delta 7.9$ and $8.1 \mathrm{ppm}$ as doublet signals for two hydrogen of pyridazine ring, and at $\delta 8.58-8.62$ and $9.08 \mathrm{ppm}$ as multiplite and single signals for $2 \mathrm{H}$ and $1 \mathrm{H}$, respectively for $\left(\left(\mathrm{NO}_{2}\right)_{2} \mathrm{C}_{6} \mathrm{H}_{3}-\right)$. Oxidation of pyridazine-3-thione (1) with sodium nitrite in acetic acid gave the bis(6-chloropyridazyl)disulfide (11) in good yield. Elemental analysis, infrared, and ${ }^{1} \mathrm{H}$ NMR data were consistent with the assigned structure. Compound (1) reacted with thiophenol or 3,6-dichloropyridazine in refluxing ethanolic sodium ethoxide to afford phenyl pyridazylthione disulfide (12) and bis(6-chloropyridazyl)sulfide 
TABLE 3: Spectral data of compounds (2-13).

\begin{tabular}{|c|c|c|c|}
\hline Comp. & IR $\mathrm{cm}^{-1}$ & ${ }^{1} \mathrm{H}$ NMR ppm & ${ }^{13} \mathrm{C}-\mathrm{NMR}$ ppm \\
\hline $2 a$ & $\begin{array}{c}3380(\mathrm{NH}), 1750(\mathrm{CO}), 1610(\mathrm{C}=\mathrm{N}) \\
1459(\mathrm{~N}-\mathrm{C}=\mathrm{S}), 1405(\mathrm{C}=\mathrm{S})\end{array}$ & $\begin{array}{l}6.1(\mathrm{~s}, 1 \mathrm{H}, \mathrm{NH} \text {, exchangeable with } \\
\left.\mathrm{D}_{2} \mathrm{O}\right), 6.9-7.6(\mathrm{~m}, 6 \mathrm{Ar} \mathrm{H})\end{array}$ & $\begin{array}{c}126.72\left(\mathrm{C}_{6}, \mathrm{C}_{9}\right), 127.45\left(\mathrm{C}_{8}\right), 130.12\left(\mathrm{C}_{4}\right), \\
130.48\left(\mathrm{C}_{3}\right), 134.76\left(\mathrm{C}_{7}\right), 154\left(\mathrm{C}_{2}\right), 162(\mathrm{C}=\mathrm{O}) .\end{array}$ \\
\hline $2 b$ & $\begin{array}{c}3410(\mathrm{NH}), 1620(\mathrm{CO}), 1595(\mathrm{C}=\mathrm{N}) \\
1451(\mathrm{~N}-\mathrm{C}=\mathrm{S}), 1411(\mathrm{C}=\mathrm{S})\end{array}$ & $\begin{array}{l}6.4(\mathrm{~s}, 1 \mathrm{H}, \mathrm{NH} \text {, exchangeable with } \\
\left.\mathrm{D}_{2} \mathrm{O}\right), 7.4-7.9(\mathrm{~m}, 4 \mathrm{H} \text { Ar H})\end{array}$ & $\begin{array}{c}114\left(\mathrm{C}_{6}\right), 123\left(\mathrm{C}_{8}\right), 131.2\left(\mathrm{C}_{4}\right), 131.6\left(\mathrm{C}_{3}\right), 1349 \\
\left(\mathrm{C}_{9}\right), 139.9\left(\mathrm{C}_{7}\right), 154\left(\mathrm{C}_{2}\right), 162(\mathrm{C}=\mathrm{O}) .\end{array}$ \\
\hline $2 c$ & $\begin{array}{l}3385(\mathrm{NH}), 1616(\mathrm{CO}), 1580(\mathrm{C}=\mathrm{N}) \\
1432(\mathrm{~N}-\mathrm{C}=\mathrm{S}), 1418(\mathrm{C}=\mathrm{S})\end{array}$ & $\begin{array}{l}6.6(\mathrm{~s}, 1 \mathrm{H}, \mathrm{NH} \text {, exchangeable with } \\
\left.\mathrm{D}_{2} \mathrm{O}\right), 7.1-7.8(\mathrm{~m}, 5 \mathrm{H}, \mathrm{Ar} \mathrm{H})\end{array}$ & $\begin{array}{c}122\left(\mathrm{C}_{8}\right), 125\left(\mathrm{C}_{6}\right), 131\left(\mathrm{C}_{4}\right), 131.8\left(\mathrm{C}_{3}\right), 133 \\
\left(\mathrm{C}_{9}\right), 138\left(\mathrm{C}_{7}\right), 153.4\left(\mathrm{C}_{2}\right) \\
162.3(\mathrm{C}=\mathrm{O})\end{array}$ \\
\hline 3 & $\begin{array}{l}3370(\mathrm{NH}), 1610(\mathrm{C}=\mathrm{N}), 1438 \\
\quad(\mathrm{~N}-\mathrm{C}=\mathrm{S}), 1401(\mathrm{C}=\mathrm{S})\end{array}$ & $\begin{array}{l}6.7(\mathrm{~s}, 1 \mathrm{H}, \mathrm{NH} \text {, exchangeable with } \\
\left.\mathrm{D}_{2} \mathrm{O}\right), 6.8-7.6(\mathrm{~m}, 6 \mathrm{H}, \mathrm{Ar} \mathrm{H})\end{array}$ & $\begin{array}{c}110.3\left(\mathrm{C}_{7}\right), 112.1\left(\mathrm{C}_{10}\right), 121.2\left(\mathrm{C}_{4}\right), 123\left(\mathrm{C}_{8}\right) \\
123\left(\mathrm{C}_{9}\right), 125.2\left(\mathrm{C}_{5}\right), 180.6\left(\mathrm{C}_{3}\right) .\end{array}$ \\
\hline 4 & $\begin{array}{c}3420(\mathrm{NH}), 3390(\mathrm{NH}), 1610(\mathrm{C}=\mathrm{N}) \\
1432(\mathrm{~N}-\mathrm{C}=\mathrm{S}), 1416(\mathrm{C}=\mathrm{S})\end{array}$ & $\begin{array}{l}6.3(\mathrm{~s}, 1 \mathrm{H}, \mathrm{NH} \text {, exchangeable with } \\
\left.\mathrm{D}_{2} \mathrm{O}\right), 6.8(\mathrm{~s}, 1 \mathrm{H}, \mathrm{NH} \text {, exchangeable } \\
\left.\text { with } \mathrm{D}_{2} \mathrm{O}\right), 6.9-7.6(\mathrm{~m}, 6 \mathrm{H}, \text { Ar H})\end{array}$ & $\begin{array}{l}116.8\left(\mathrm{C}_{5} \& \mathrm{C}_{8} \text { benzotriazine }\right), 120.4\left(\mathrm{C}_{6} \&\right. \\
\left.\mathrm{C}_{7} \text {, benzotriazine }\right), 132.4\left(\mathrm{C}_{4}, \text { pyridazine }\right), \\
\text { 134.1- }\left(\mathrm{C}_{5}, \text { pyridazine }\right), 151\left(\mathrm{C}_{6}\right), 154.1\left(\mathrm{C}_{3}\right) .\end{array}$ \\
\hline 5 & $\begin{array}{c}3310(\mathrm{NH}), 1660(\mathrm{CO}), 1605(\mathrm{C}=\mathrm{N}) \\
1440(\mathrm{~N}-\mathrm{C}=\mathrm{S}), 1413(\mathrm{C}=\mathrm{S})\end{array}$ & $\begin{array}{c}2.95\left(\mathrm{~d}, 1 \mathrm{H},-\mathrm{CH}_{2}\right), 3.15\left(\mathrm{~d}, 1 \mathrm{H},-\mathrm{CH}_{2}\right) \\
3.85(\mathrm{~d}, 1 \mathrm{H},-\mathrm{CH}), 6.83(\mathrm{~s}, 1 \mathrm{H}, \mathrm{NH} \\
\left.\text { exchangeable with } \mathrm{D}_{2} \mathrm{O}\right), 7.41-7.96(\mathrm{~m}, \\
\text { 7H, Ar H). }\end{array}$ & $\begin{array}{c}40.1\left(\mathrm{CH}_{2}\right), 72.4\left(\mathrm{C}_{4}, \text { imidazole }\right), 125.1,127.3 \text {, } \\
128(\mathrm{Ph}-\mathrm{C}), 130\left(\mathrm{C}_{5}, \text { pyridazine }\right), 131.3\left(\mathrm{C}_{4} \text {, }\right. \\
\text { pyridazine }), 152.3\left(\mathrm{C}_{6}, \text { pyridazine }\right), 154.2 \\
\left(\mathrm{C}_{3}, \text { pyridazine }\right), 174.2(\mathrm{C}=\mathrm{O}) .\end{array}$ \\
\hline 6 & $\begin{array}{l}3427-3409\left(\mathrm{NH}_{2}\right), 3316(\mathrm{NH}), 1580 \\
(\mathrm{C}=\mathrm{N}), 1444(\mathrm{NC}=\mathrm{S}), 1408(\mathrm{C}=\mathrm{S})\end{array}$ & $\begin{array}{c}6.29\left(\mathrm{~s}, 2 \mathrm{H}, \mathrm{NH}_{2} \text {, exchangeable with }\right. \\
\left.\mathrm{D}_{2} \mathrm{O}\right), 6.45(\mathrm{~s}, 1 \mathrm{H}, \mathrm{NH}), 6.81-6.93(\mathrm{~m}, \\
2 \mathrm{H}, \mathrm{Ar} \mathrm{H}) \\
\end{array}$ & $\begin{array}{c}122.1\left(\mathrm{C}_{4}, \text { pyridazine }\right), 125.3\left(\mathrm{C}_{5}, \text { pyridazine }\right), \\
137.9\left(\mathrm{C}_{3}, \text { triazole }\right), 141.6\left(\mathrm{C}_{6}, \text { pyridazine }\right), \\
179.3\left(\mathrm{C}_{3}, \mathrm{p} \text {-yridazine }\right) .\end{array}$ \\
\hline $7 \mathbf{a}$ & $\begin{array}{l}3357(\mathrm{NH}), 1600(\mathrm{C}=\mathrm{N}), 1425 \\
\quad(\mathrm{~N}-\mathrm{C}=\mathrm{S}), 1405(\mathrm{C}=\mathrm{S})\end{array}$ & $\begin{array}{l}6.52(\mathrm{~s}, 1 \mathrm{H}, \mathrm{NH} \text {, exchangeable with } \\
\left.\mathrm{D}_{2} \mathrm{O}\right), 6.81-7.84(\mathrm{~m}, 6 \mathrm{H}, \mathrm{Ar} \mathrm{H})\end{array}$ & $\begin{array}{c}119.9\left(\mathrm{C}_{4} \text {, pyridazine }\right), 122.2\left(\mathrm{C}_{5} \text {, pyridazine }\right) \text {, } \\
126.1\left(\mathrm{C}_{1}, \text { phenyl }\right), 126.7\left(\mathrm{C}_{2} \& \mathrm{C}_{6}, \text { phenyl }\right) \\
129.1\left(\mathrm{C}_{3} \& \mathrm{C}_{5}, \text { phenyl }\right), 132.4(\mathrm{C}-\mathrm{Cl}), 179.1 \\
\left(\mathrm{C}_{3} \text {, pyridazine }\right) \text {. }\end{array}$ \\
\hline $7 \mathbf{b}$ & $\begin{array}{l}3349(\mathrm{NH}), 1606(\mathrm{C}=\mathrm{N}), 1432 \\
\quad(\mathrm{~N}-\mathrm{C}=\mathrm{S}), 1418(\mathrm{C}=\mathrm{S})\end{array}$ & $\begin{array}{l}6.48(\mathrm{~s}, 1 \mathrm{H}, \mathrm{NH}, \text { exchangeable with } \\
\left.\mathrm{D}_{2} \mathrm{O}\right), 6.76-7.59(\mathrm{~m}, 7 \mathrm{H}, \text { Ar H) }\end{array}$ & $\begin{array}{c}120.1\left(\mathrm{C}_{4}, \text { pyridazine }\right), 122.5\left(\mathrm{C}_{5}, \text { pyridazine }\right), \\
127.2\left(\mathrm{C}_{2} \& \mathrm{C}_{6}, \text { phenyl }\right) 129.2\left(\mathrm{C}_{3} \& \mathrm{C}_{5},\right. \\
\text { phenyl }), 130.6\left(\mathrm{C}_{1}, \text { phenyl }\right), 130.9\left(\mathrm{C}_{4},\right. \\
\text { phenyl }), 139.8\left(\mathrm{C}_{3}, \text { triazole }\right), 180.1\left(\mathrm{C}_{3},\right. \\
\text { pyridazine })\end{array}$ \\
\hline $8 \mathbf{a}$ & $\begin{array}{c}3420(\mathrm{NH}), 3391(\mathrm{NH}), 1610(\mathrm{C}=\mathrm{N}) \\
1429(\mathrm{~N}-\mathrm{C}=\mathrm{S}), 1408(\mathrm{C}=\mathrm{S})\end{array}$ & $\begin{array}{l}2.14\left(\mathrm{~s}, 3 \mathrm{H}, \mathrm{CH}_{3}\right), 6.42(\mathrm{~s}, 1 \mathrm{H}, \mathrm{NH} \\
\left.\text { exchangeable with } \mathrm{D}_{2} \mathrm{O}\right), 6.9-7.7(\mathrm{~m}, \\
\text { 7H, Ar H), } 10.14(\mathrm{~s}, 1 \mathrm{H}, \mathrm{NH} \\
\left.\text { exchangeable with } \mathrm{D}_{2} \mathrm{O}\right)\end{array}$ & $\begin{array}{c}25.4\left(\mathrm{CH}_{3}\right), 81.5\left(\mathrm{C}_{3} \text {, triazole }\right), 126\left(\mathrm{C}_{4},\right. \\
\text { phenyl }), 126.9\left(\mathrm{C}_{2} \& \mathrm{C}_{6} \text {, phenyl }\right), 128\left(\mathrm{C}_{3} \&\right. \\
\left.\mathrm{C}_{5}, \text { phenyl }\right), 129.8\left(\mathrm{C}_{4}, \text { pyridazine }\right), 134.2 \\
\left(\mathrm{C}_{5} \text {, pyridazine }\right), 141.2\left(\mathrm{C}_{1} \text {, phenyl }\right), 150.1 \\
\left(\mathrm{C}_{3}, \text { pyridazine }\right) .\end{array}$ \\
\hline $8 b$ & $\begin{array}{c}3406(\mathrm{NH}), 3360(\mathrm{NH}), 1590 \\
(\mathrm{C}=\mathrm{N}), 1437(\mathrm{~N}-\mathrm{C}=\mathrm{S}), 1410(\mathrm{C}=\mathrm{S}) .\end{array}$ & $\begin{array}{c}2.23\left(\mathrm{~s}, 3 \mathrm{H}, \mathrm{CH}_{3}\right), 6.56(\mathrm{~s}, 1 \mathrm{H}, \mathrm{NH} \\
\left.\mathrm{D}_{2} \mathrm{O} \text {-exchangeable }\right), 6.8-7.7(\mathrm{~m}, 6 \mathrm{H} \\
\text { Ar } \mathrm{H}), 10.67(\mathrm{~s}, 1 \mathrm{H}, \mathrm{NH}, \text { exchangeable } \\
\left.\text { with } \mathrm{D}_{2} \mathrm{O}\right)\end{array}$ & $\begin{array}{c}25.8\left(\mathrm{CH}_{3}\right), 83.2\left(\mathrm{C}_{3} \text {, triazole }\right), 122\left(\mathrm{C}_{4},\right. \\
\text { phenyl }), 123.5\left(\mathrm{C}_{2} \text {, phenyl }\right), 129.1\left(\mathrm{C}_{5},\right. \\
\text { phenyl }), 129.8\left(\mathrm{C}_{4}, \text { pyridazine }\right), 131.2\left(\mathrm{C}_{4} \text {, }\right. \\
\text { pyridazine }), 133.2\left(\mathrm{C}_{6}, \text { phenyl }\right), 136.2\left(\mathrm{C}_{5} \text {, }\right. \\
\text { pyridazine }), 143.2\left(\mathrm{C}_{1}, \text { phenyl }\right), 148.2 \\
\left(\mathrm{C}-\mathrm{NO}_{2}\right), 152.3\left(\mathrm{C}_{3}, \text { pyridazine }\right) .\end{array}$ \\
\hline 9 & $\begin{aligned} 3360(\mathrm{NH}), & 1572(\mathrm{C}=\mathrm{N}), 1417(\mathrm{C}=\mathrm{S}) \\
& 1345(\mathrm{~S}=\mathrm{O})\end{aligned}$ & $\begin{array}{c}2.99\left(\mathrm{~s}, 3 \mathrm{H}, \mathrm{CH}_{3}\right), 6.5(\mathrm{~s}, 1 \mathrm{H}, \mathrm{NH} \\
\left.\text { exchangeable with } \mathrm{D}_{2} \mathrm{O}\right), 6.89-7.92(\mathrm{~m}, \\
6 \mathrm{H}, \mathrm{Ar} \mathrm{H})\end{array}$ & $\begin{array}{l}22.1\left(\mathrm{CH}_{3}\right), 123.3\left(\mathrm{C}_{1}, \text { phenyl }\right), 128.1\left(\mathrm{C}_{3} \&\right. \\
\left.\mathrm{C}_{5}, \text { phenyl }\right), 129.2\left(\mathrm{C}_{5}, \text { pyridazine }\right), 129.8 \\
\left(\mathrm{C}_{4}, \text { pyridazine }\right), 130.4\left(\mathrm{C}_{2} \& \mathrm{C}_{6} \text {, phenyl }\right), \\
132.6\left(\mathrm{C}_{4}, \text { phenyl }\right), 154.1\left(\mathrm{C}_{3}, \text { pyridazine }\right) .\end{array}$ \\
\hline 10 & $\begin{array}{c}1605(\mathrm{C}=\mathrm{N}), 1530-1380\left(\mathrm{NO}_{2}\right), 1340 \\
(\mathrm{C}-\mathrm{S}-) .\end{array}$ & $\begin{array}{c}7.9(\mathrm{~d}, 1 \mathrm{H}, \mathrm{CH}), 8.1(\mathrm{~d}, 1 \mathrm{H}, \mathrm{CH}) \\
8.58-8.62\left(\mathrm{~m}, 2 \mathrm{H},\left(\mathrm{NO}_{2}\right)_{2} \mathrm{C}_{6} \mathrm{H}_{3}-\right), 9.08 \\
\left(\mathrm{~s}, 1 \mathrm{H},\left(\mathrm{NO}_{2}\right)_{2} \mathrm{C}_{6} \mathrm{H}_{3}-\right)\end{array}$ & $\begin{array}{c}123.6\left(\mathrm{C}_{5}, \text { pyridazine }\right), 124.8\left(\mathrm{C}_{3}, \text { phenyl }\right), \\
129.1\left(\mathrm{C}_{5}, \text { phenyl }\right), 129.3\left(\mathrm{C}_{4}, \text { pyridazine }\right), \\
143.2\left(\mathrm{C}_{4}, \text { phenyl }\right), 145.9\left(\mathrm{C}_{2}, \text { phenyl }\right), 152.2 \\
(\mathrm{C}-\mathrm{Cl}), 178.2\left(\mathrm{C}_{6}, \text { pyridazine }\right) . \\
\end{array}$ \\
\hline 11 & $1620(\mathrm{C}=\mathrm{N})$ & $7.9(\mathrm{~d}, 2 \mathrm{H}, 2 \mathrm{CH}), 8.0(\mathrm{~d}, 2 \mathrm{H}, 2 \mathrm{CH})$ & $\begin{array}{c}124.2\left(2 \mathrm{C}_{5}\right), 125.4\left(2 \mathrm{C}_{4}\right), 152.2(2 \mathrm{C}-\mathrm{Cl}), 178.1 \\
\left(2 \mathrm{C}_{6}\right) .\end{array}$ \\
\hline 12 & $\begin{array}{c}3340(\mathrm{NH}), 1600(\mathrm{C}=\mathrm{N}), 1273 \\
(\mathrm{C}-\mathrm{S}-)\end{array}$ & $\begin{array}{l}6.35(\mathrm{~s}, 1 \mathrm{H}, \mathrm{NH}, \text { exchangeable with } \\
\left.\mathrm{D}_{2} \mathrm{O}\right), 6.81-7.98(\mathrm{~m}, 7 \mathrm{H}, \text { Ar H) }\end{array}$ & $\begin{array}{c}120.3\left(\mathrm{C}_{4} \& \mathrm{C}_{5}, \text { pyridazine }\right), 122.5\left(\mathrm{C}_{4},\right. \\
\text { phenyl }), 127.1\left(\mathrm{C}_{2} \& \mathrm{C}_{6} \text {, phenyl }\right), 128.2\left(\mathrm{C}_{3} \&\right. \\
\left.\mathrm{C}_{5} \text {, phenyl }\right), 134.2\left(\mathrm{C}_{1} \text {, phenyl }\right), 177.2(2 \mathrm{C}-\mathrm{S}) .\end{array}$ \\
\hline 13 & $1580(\mathrm{C}=\mathrm{N})$ & $7.99(\mathrm{~d}, 2 \mathrm{H}, 2 \mathrm{CH}), 8.87(\mathrm{~d}, 2 \mathrm{H}, 2 \mathrm{CH})$ & $\begin{array}{c}122.1\left(2 \mathrm{C}_{5}\right), 126.3\left(2 \mathrm{C}_{4}\right), 152.6(2 \mathrm{C}-\mathrm{Cl}), 178 \\
(2 \mathrm{C}-\mathrm{S}) .\end{array}$ \\
\hline
\end{tabular}


(13), respectively (Scheme 3). Elemental analysis, infrared, ${ }^{1} \mathrm{H}$ $\mathrm{NMR}$, and ${ }^{13} \mathrm{C}$ NMR data of (12) and (13) are given in Tables 2 and 3.

3.1. Antimicrobial Activity. The antimicrobial activity of the synthesized compounds has been evaluated by filter paper disc method [21]. The synthesized compounds have been tested for their antibacterial activity against Staphylococcus aureus ATCC6538P, Bacillus subtilis ATCC6633, Pseudomonas aurignosa ATCC9027, and Echerichia coli ATCC8739 and antifungal activity against Candida albicans ATCC2091, Aspergillus niger, at a concentration of $500 \mu \mathrm{g} / \mathrm{mL}$ in DMF. Nutrient agar and potato dextrose agars were used to culture the bacteria and fungi, respectively. The plates were inculcated by the bacteria or fungi and incubated for $24 \mathrm{~h}$ at $37^{\circ} \mathrm{C}$ for bacteria and for $72 \mathrm{~h}$ at $27^{\circ} \mathrm{C}$ for fungi and then the inhibition zones of microbial growth surrounding the filter paper disc $(5 \mathrm{~mm})$ were measured in millimeters. Ampicillin and mycostatin, at a concentration $500 \mu \mathrm{g} / \mathrm{mL}$, were used as standard against bacteria and fungyi, respectively. Test results are shown in Table 1. From the data, it is clear that compounds (2a), (4) and (9) possess high activity, while compounds (3), (6), (8b), and (11) possess moderate activity against grampositive strains. As far as gram-negative microorganisms are concerned, compounds (2a), (9), and (11) showed high activity while compounds (3), (7a), and (12) display moderate activity. Compounds (9) and (12) also exerted high activity while compounds (2a), (3), (4), and (8b) have moderate activity against fungi.

\section{Conclusion}

6-Chloropyridazin-3(2H)-thione (1) has been shown to be a useful building block for the synthesis of some dropyridazino[6,1-b]quinazolin-10-ones, 1,2,4-benzotriazinopyrid-azinethione, imidazo[1,2-b]pyridazinethione, 1,2,4triazolo[4,3-b]pyridazinethione, and disulfide. The structure of all newly synthesized compounds was established from their spectral data and elemental analysis. Some of these compounds possess a highly response against gram-positive and gram-negative bacteria as well as fungi.

\section{Acknowledgment}

The authors extend their appreciation to the Deanship of Scientific Research at King Saud University for funding this paper through the research group Project no. RGP-VPP-133.

\section{References}

[1] N. G. Kandile, M. I. Mohamed, H. Zaky, and H. M. Mohamed, "Novel pyridazine derivatives: synthesis and antimicrobial activity evaluation," European Journal of Medicinal Chemistry, vol. 44, no. 5, pp. 1989-1996, 2009.

[2] M. Asif, A. Singh, and L. Ratnakar, "Antimicrobial agents: brief study of pyridazine derivatives against some phathogenic microrganisms," Journal of Pharmacy Research, vol. 4, no. 3, pp. 664-667, 2011.
[3] A. Benmoussa, J. El harti, M. Ansar et al., "Synthesis and antimicrobial properties of some pyridazin-3-thiones derivatives," International Journal of PharmTech Research, vol. 4, no. 4, pp. 1591-1594, 2012.

[4] I. I. Mangalagiu, "Recent achievements in the chemistry of 1,2diazines," Current Organic Chemistry, vol. 15, no. 5, pp. 730-752, 2011.

[5] D. Mantu, M. C. Luca, C. Moldoveanu, G. Zbancioc, and I. I. Mangalagiu, "Synthesis and antituberculosis activity of some new pyridazine derivatives. Part II," European Journal of Medicinal Chemistry, vol. 45, no. 11, pp. 5164-5168, 2010.

[6] R. M. Butnariu and I. I. Mangalagiu, "New pyridazine derivatives: synthesis, chemistry and biological activity," Bioorganic and Medicinal Chemistry, vol. 17, no. 7, pp. 2823-2829, 2009.

[7] G. Drochioiu, V. Sunel, C. Oniscu, C. Basu, and M. Murariu, "The breakdown of plant biostructure followed by amino acids determination," Romanian Biotechnological Letters, vol. 6, no. 2, pp. 155-165, 2001.

[8] R. M. Butnariu, M. D. Caprosu, V. Bejan et al., "Pyridazine and phthalazine derivatives with potential antimicrobial activity," Journal of Heterocyclic Chemistry, vol. 44, no. 5, pp. 1149-1152, 2007.

[9] S. Dima, M. Caprosu, M. Ungureanu, G. Grosu, and M. Petrovanu, "Antimicrobial and antifungical activity of some new 1-methyl-phtalazinium compounds," Annales Pharmaceutiques Francaises, vol. 57, no. 5, pp. 415-416, 1999.

[10] X. Han, Y. H. Hong, Z. H. U. You Quan, Z. X. Mao LIU Bim, H. U. Fang Zhong, and Y. H. Zheng, "Synthesis and herbicidal activities of novel 4-(3-trifluoromethylphenyl)-2H-pyridazin3-one derivatives," Science China Chemistry, vol. 53, no. 1, pp. 157-166, 2010.

[11] H. M. Refaat, O. M. Khalil, and H. H. Kadry, "Synthesis and antiinflammatory activity of certain piperazinylthienylpyridazine derivatives," Archives of Pharmacal Research, vol. 30, no. 7, pp. 803-811, 2007.

[12] C. Liljebris, J. Martinsson, L. Tedenborg et al., "Synthesis and biological activity of a novel class of pyridazine analogues as non-competitive reversible inhibitors of protein tyrosine phosphatase 1B (PTP1B)," Bioorganic and Medicinal Chemistry, vol. 10, no. 10, pp. 3197-3212, 2002.

[13] R. B. Tucaliuc, I. M. Risca, G. Drochioiu, and I. Mangalagiu, "Biological effect of some new pyridazine derivatives on wheat in germination experiments," Romanian Biotechnological Letters, vol. 13, no. 4, pp. 3837-3842, 2008.

[14] T. Ishikawa, Y. Iizawa, K. Okonogi, and A. Miyake, "Studies on Anti-MRSA parenteral cephalosporins I. Synthesis and antibacterial activity of 7," Journal of Antibiotics, vol. 53, no. 10, pp. 1053-1070, 2000.

[15] C. Almansa, A. F. De Arriba, F. L. Cavalcanti et al., "Synthesis and SAR of a new series of COX-2-selective inhibitors: pyrazolo[1,5-a]pyrimidines," Journal of Medicinal Chemistry, vol. 44, no. 3, pp. 350-361, 2001.

[16] G. B. Barlin, L. P. Davies, and P. W. Harrison, "Imidazo[1,2b]pyridazines. XXI. Syntheses of some 3-acylaminomethyl-6(chloro and iodo)-2-(substituted phenyl)-imidazo[1,2-b]pyridazines and -imidazo[1,2-a]pyridines and their interaction with central and mitochondrial benzodiazepine," Australian Journal of Chemistry, vol. 50, no. 1, pp. 61-68, 1997.

[17] H. M. Faidallah, K. A. Khan, and M. S. I. Makki, "Synthesis and biological evaluation of new fused isoxazolo[4,5-d]pyridazine derivatives," Journal of the Chinese Chemical Society, vol. 58, no. 2, pp. 191-198, 2011. 
[18] N. F. Abd El-Ghaffar, M. K. Mohamed, M. S. Kadah, A. M. Radwan, G. H. Said, and S. N. Abd el Al, "Synthesis and antitumor activities of some new pyridazinones containing the 2phenyl-1H-indolyl moiety," Journal of Chemical and Pharmaceutical Research, vol. 3, no. 3, pp. 248-259, 2011.

[19] M. H. Sherif, G. A. Ahmed, A. A. Elbahnasawy, and E. O. Helal, "Chemical studies on 3,6-dichloropyridazine," Journal of American Science, vol. 6, no. 11, pp. 570-574, 2010.

[20] N. M. Abd El-Salam, Z . Y. Al-Shoaibi, and G. A. Ahmed, "Chemical studies on 3,6-dichloropyridazine (part 2)," EJournal of Chemistry, vol. 8, no. 4, pp. 1944-1950, 2011.

[21] Y. L. Nene and P. N. Thapliyal, Fungicides in Plant Disease Control, IBH, New Delhi, India, 1982. 

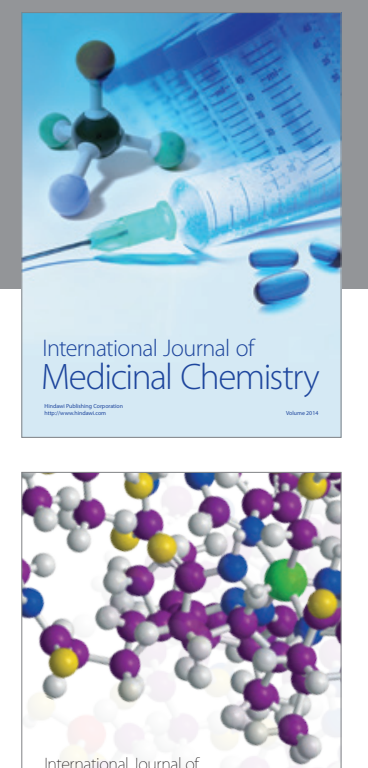

\section{Carbohydrate} Chemistry

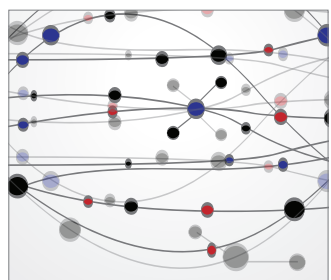

The Scientific World Journal
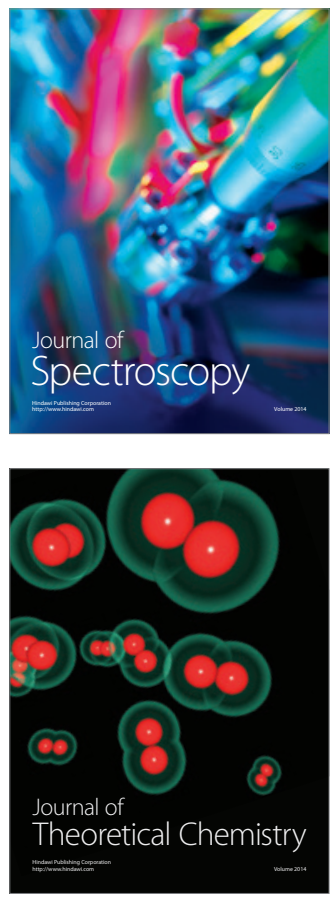
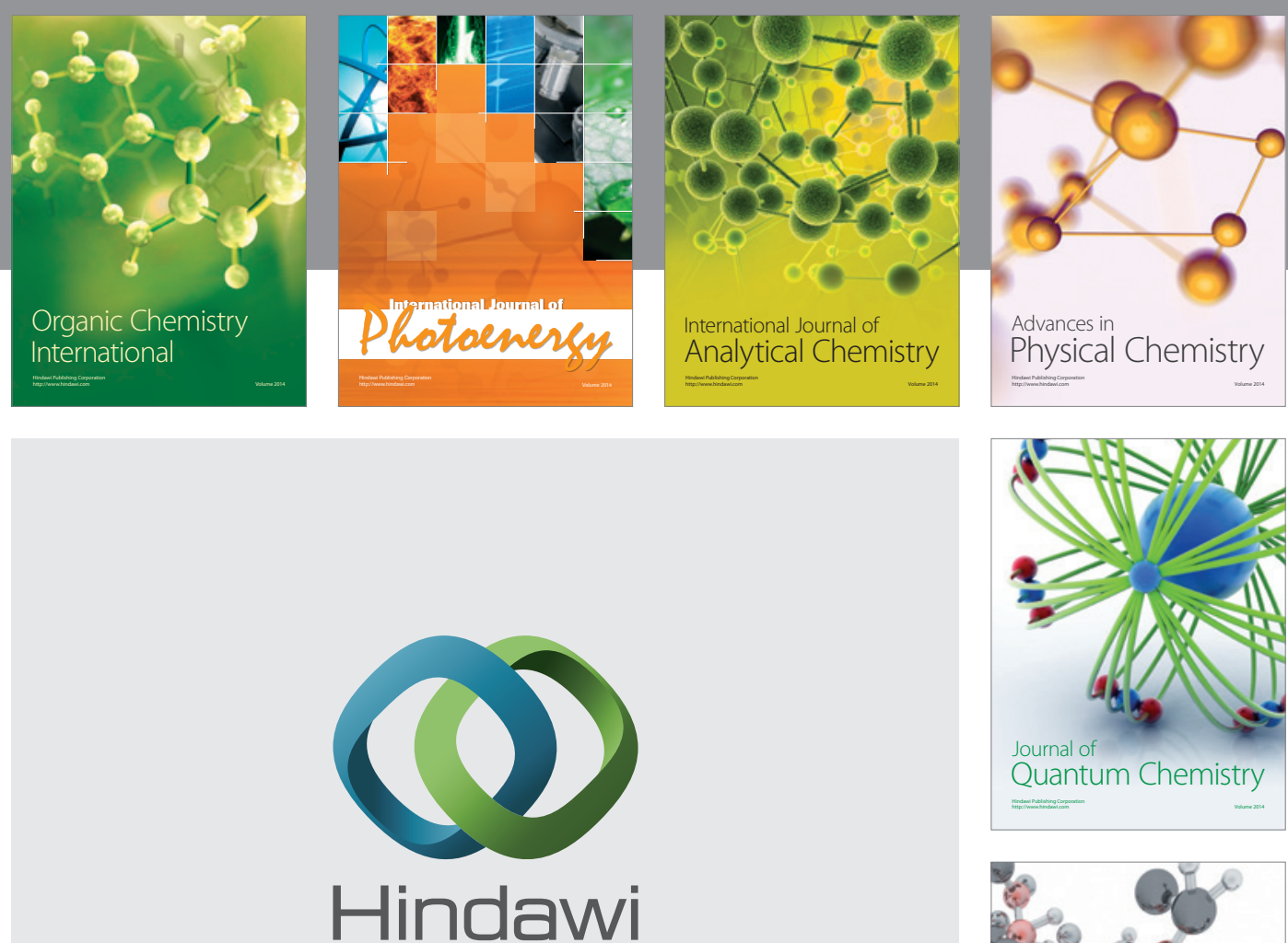

Submit your manuscripts at

http://www.hindawi.com

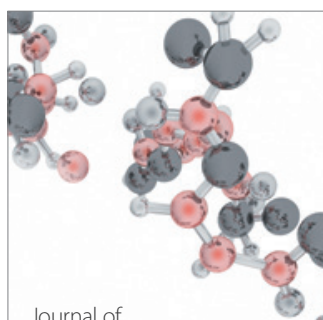

Analytical Methods

in Chemistry

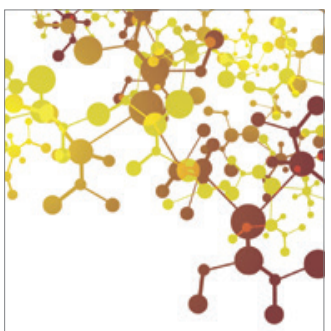

Journal of

Applied Chemistry

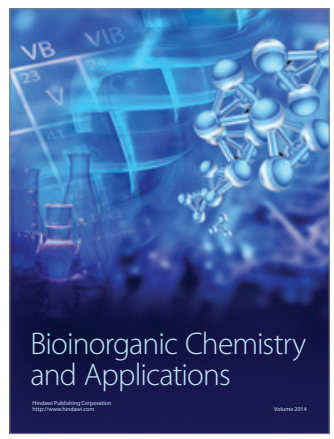

Inorganic Chemistry
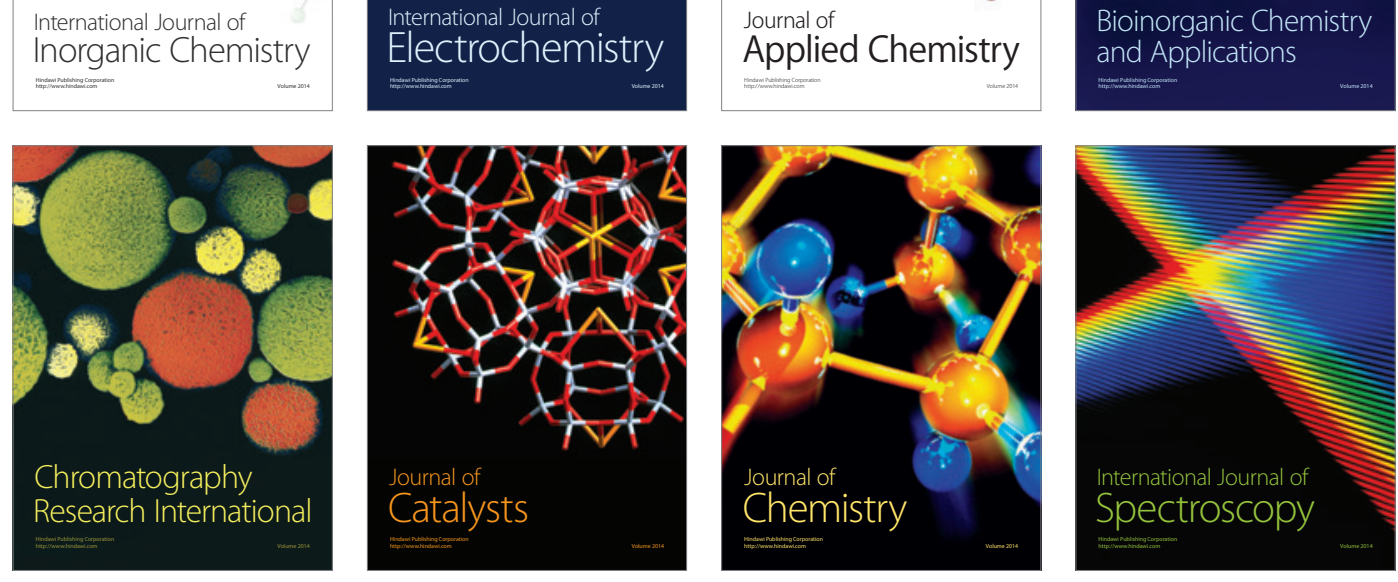